\title{
Prevalence of chronic thyroiditis in a cohort of patients with papillary carcinoma of thyroid
}

N. M. Garusinghe ${ }^{1}$, R. K. H. Priyangika ${ }^{1}$ and A. U. Kiriwandeniya ${ }^{2}$

Department of Allied Health Sciences, Faculty of Medical Sciences, University of Sri Jayewardenepura, Sri Lanka ${ }^{1}$

Department of Pathology, Faculty of Medical Sciences, University of Sri Jayewardenepura, Sri Lanka ${ }^{2}$

DOI: http://doi.org/10.4038/jdp.v11i2.7714

\section{Introduction}

Chronic thyroiditis (CT) is known to be associated with papillary carcinoma of thyroid (PCT). The aim of this study was to identify the prevalence of chronic thyroiditis in a cohort of patients with papillary carcinoma of thyroid and to assess the pre-surgical awareness of existence of chronic thyroiditis.

\section{Methodology}

Patients who underwent thyroidectomy from August 2013 to August 2015 at Colombo South Teaching Hospital for PCT were included in the study. Presence of a presurgical diagnosis of CT was assessed by available details in request forms, histopathological and cytopathological reports and/or by direct telephone interviews.

\section{Results}

The total study population was 114 with a female preponderance $(94 / 114,82.5 \%)$. The mean age was 41.52 years and the highest occurrence was in $30-40$ years age group. Out of 114 PCT patients $63.2 \%$ (72/114) were associated with background CT. Of these 32 (44.4\%) did not have a pre-surgical diagnosis of CT.

\section{Discussion}

Two thirds (2/3) of our study population with PCT had associated CT. Majority of them were not aware of CT until after the surgical resection of the gland. However, this study may under-estimate the prevalence of background CT in PCT in both histological and cytological assessments since the study is a review of reports rather than of slides.

\section{Conclusion}

Results of this preliminary study emphasize the need for further studies to establish the percentage of papillary carcinoma of thyroid associated with chronic thyroiditis in the Sri Lankan population. This may help in assessing the feasibility of a population screening programme. 